\title{
Biochemical Characterization of the Actions of Ghemotherapeutic Agents
}

\author{
4. TIME-RELATIONSHIPS BETWEEN METABOLIC AND \\ GROWTH INHIBITIONS BY PANTOYLTAURINE
}

\begin{abstract}
By H. McILWAIN (Leverhulme Research Fellow), Department of Bacterial Chemistry
(Medical Research Council), The University, Sheffield
\end{abstract}

(Received 17 May 1945)

The manner in which pantoyltaurine influenced the course of streptococcal growth, when relatively small inocula of the non-proliferating bacteria were added to growth media already containing the inhibitor, was described previously (McIlwain,' 1944). In chemotherapeutic practice, however, such a drug is commonly administered at a time when the infecting micro-organism is already growing. The addition of sulphonamides to growing bacterial cultures in vitro, to simulate administration of this type, has usually shown that the action of the drugs upon growth follows a latent period of an hour or more (see Henry, 1943). Similar characters have now been found in the action of pantoyltaurine upon streptococcal growth and an attempt has been made to understand the compound's delayed action. In this attempt the promptness of action of pantoyltaurine on a process of pantothenate-inactivation (Mchwain \& Hughes, 1944) has been examined, as inhibition of this process closely parallels the majority of the growth effects of the inhibitor, and may be causally related to them.

\section{EXPERIMENTAL}

The majority of the experiments were carried out with a $\beta$-haemolytic streptococcus of group $G$, recently passaged through mice and maintained in blood broth. The Richards (group A) streptococcus and strains of Corynebacterium diphtheriae were also examined. Growth of the streptococci was followed anaerobically at $37^{\circ}$, by the manometric methods described previously (McIlwain, 1944), in a medium containing a casein hydrolysate, glucose, defined quantities of pantothenate, other growth factors, and buffered at $\mathrm{pH} 7 \cdot 6$ by bicarbonate and a gas mixture of $5 \% \mathrm{CO}_{2}$ in $\mathrm{N}_{2}$. Evolution of gas was measured; in growing cultures the rate of evolution increased with time and logarithms of the rate (in $\mu \mathrm{l} . / 15 \mathrm{~min}$.) were plotted against time to produce the growth curves of Figs. 2-4. Additions of reagents during growth were made from the side-arms of Warburg vessels.

Reaction with pantothenate was also carried out anaerobically at $37^{\circ}$ in Warburg vessels and in the presence of glucose, bicarbonate, $\mathrm{Mg}^{++}$, yeast adenylic acid and in some cases (see Table 1) of a casein hydrolysate, but in the absence of other factors necessary for growth of the organisms. Preparation of the bacterial suspensions, assay of pantothenate, and other details followed the description of McIlwain \& Hughes (1945).

\section{RESULTS}

\section{Promptness of action of pantoyltaurine on pantothenate inactivation}

Kinetics of pantothenate inactivation by haemolytic streptococci. The pantothenate content of a solution in which streptococci could inactivate pantothenate at $37^{\circ}$ was not appreciably altered if it was mixed with streptococci at that temperature and then immediately cooled to $0^{\circ}$ (Table 1). When cooled

Table 1. Effects of cooling, and of inhibitors, on pantothenate inactivation by bacteria

Vessels contained the organisms in a side-arm, and in the main compartment: glucose, $200 \mu \mathrm{mol}$.; $\mathrm{MgSO}_{4}, 0.5 \mu \mathrm{mol}$; yeast adenylic acid, $0.7 \mu \mathrm{mol}$; $\mathrm{KH}_{2} \mathrm{PO}_{4}, 10 \mu \mathrm{mol}$.; $\mathrm{NaHCO}_{3}$, $150 \mu \mathrm{mol}$; pantothenate, $20 \mathrm{~m} \mu \mathrm{mol}$. and, in (B) only, hydrolyzed casein (75 mg.), and were equilibrated with $\mathrm{N}_{2}+5 \% \mathrm{CO}_{2}$. Fluid volume: $3 \cdot 2 \mathrm{ml}$. Cooling was to $0^{\circ}$. The bacteria were separated by centrifuging or filtering, and pantothenate assayed in the supernatant fluids.

$$
\begin{array}{cc}
\text { Operations following the mixing } & \begin{array}{c}
\text { Panto- } \\
\text { thenate } \\
\text { of bacteria and pantothenate- } \\
\text { containing solutions at } 37^{\circ}
\end{array} \\
\text { inactivated }
\end{array}
$$

(A) Group G streptococcus; 5.1 mg. dry weight

Cooled immediately

Cooled after 20 min. at $37^{\circ}$

Cooled after $55 \mathrm{~min}$. at $37^{\circ}$

Pantoyltaurine $(2 \mu \mathrm{mol}$.) added $20 \mathrm{~min}$. after $\quad \mathbf{1 . 6}$

mixing; left at $37^{\circ}$ for further $35 \mathrm{~min}$.

Pantoyltaurine $(0 \cdot 2 \mu \mathrm{mol}$.) added $20 \mathrm{~min}$.

after mixing; left at $37^{\circ}$ for further $35 \mathrm{~min}$.

(B) Group G streptococcus; 4.9 mg. dry weight

Cooled 20 min. after mixing $\quad 2.65$

Iodoacetate $(6 \mu \mathrm{mol}$.) added $20 \mathrm{~min}$. after $\mathbf{2 \cdot 6}$

mixing

Cooled 50 min. after mixing

Pantoyltauin (2um

mixing; left at $37^{\circ}$ for further $30 \mathrm{~min}$.

Pantoyltaurine $(20 \mu \mathrm{mol}$.) added $20 \mathrm{~min}$. after $\quad 2.75$

mixing; left at $37^{\circ}$ for further $30 \mathrm{~min}$.

(C) C. diphtheriae (mitis); $3.9 \mathrm{mg}$. dry weight

Cooled $25 \mathrm{~min}$. after mixing $\quad 1.4$

Cooled 105 min. after mixing

$4 \cdot 8$

Pantoyltaurine $(5 \mu \mathrm{mol}$.) added $25 \mathrm{~min}$. after $\quad \mathbf{1 . 5 5}$ mixing; left at $37^{\circ}$ for further $80 \mathrm{~min}$. 
to $0^{\circ}$ at intervals, after mixing and keeping at $37^{\circ}$, the disappearance of pantothenate was progressive (Fig. 1) and not unusually large in the first

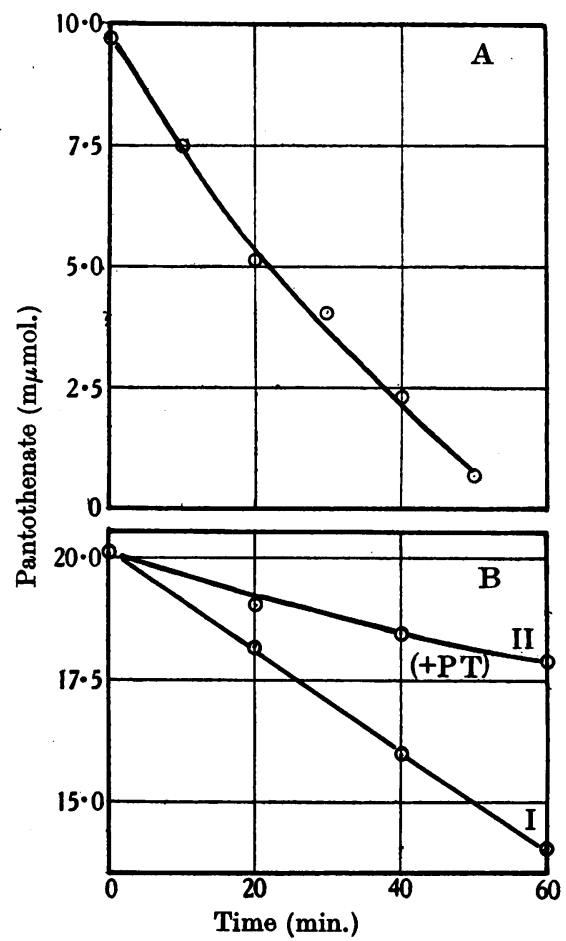

Fig. 1. Kinetics of pantothenate inactivation by a group $\mathbf{G}$ haemolytic streptococcus. (A) Reaction mixture of McIlwain \& Hughes (1945) with glucose $(200 \mu \mathrm{mol}$.) and pantothenate (10 m $\mu \mathrm{mol}$.). Organisms (dry weight $\mathbf{7 \cdot 6} \mathbf{~ m g}$.) were added after equilibration at a time taken as zero in the figure. Vessels were cooled in ice at the intervals indicated. (B) The vessels of set I contained the above reaction mixture with pantothenate, $20 \mathrm{~m} \mu \mathrm{mol}$. and those of set II with pantothenate, $20 \mathrm{~m} \mu \mathrm{mol}$. and pantoyltaurine (PT), $200 \mathrm{~m} \mu \mathrm{mol}$. Organisms: $2.65 \mathrm{mg}$. (dry weight)/vessel.

period. A disproportionately large inactivation of pantothenate in the initial period would be expected if a reaction continued after cooling, or if pantothenate inactivation was not a progressive metabolic process. The small quantities of pantothenate which were involved in the present experiments made it especially necessary to establish this point. These quantities - about $5 \mu \mathrm{g}$. of pantothenate with the $5 \mathrm{mg}$. dry weight of bacteria of a typical experiment-are, however, much greater than the pantothenate of the bacteria themselves, which in several cases examined approximated to $0.06 \mu \mathrm{g}$.; gain or loss of pantothenate by the bacterial cells was very small in comparison with the changes in the pantothenate of the solutions which are reported in the present account.
Table 1 shows also that when a reaction mixture was cooled at the same time as an otherwise similar one was mixed with iodoacetate, the pantothenate inactivated in the two cases was the same. The effect of iodoacetate on acid production from glucose which, with the present reaction mixture, was essential to pantothenate inactivation, was an immediate inhibition. These experiments show that the effect of cooling upon pantothenate metabolism is to inhibit it very rapidly. This operation has been used as standard in judging the promptness of action of pantoyltaurine.

Fig. 1 shows also the progress of pantothenate inactivation with time, over a range of pantothenate concentrations $(6 \cdot 6-0.3 \mathrm{~m} \mu \mathrm{mol} . / \mathrm{ml}$.) which includes all the pantothenate concentrations studied in the present experiments, as well as those of the blood of animals in which experimental chemotherapy with pantoyltaurine was carried out (McIlwain \& Hawking, 1943). The pantothenate inactivation is approximately directly proportional to time but falls off slightly at the lower pantothenate concentrations. In the calculations of the following paragraph, which concern only the higher pantothenate concentrations, the relationship is taken as a linear one; such a relationship also holds over the narrower range of concentrations of Fig. 1B, during pantothenate inactivation which is partially inhibited by the addition of pantoyltaurine to the initial reaction mixture.

Addition of pantoyltaurine during pantothenate metabolism. In experiments of Table 1, pantoyltaurine was added to reaction mixtures containing pantothenate at the same time as duplicate mixtures were cooled to $0^{\circ}$ or mixed with iodoacetate. Uninhibited controls, and the mixtures containing pantoyltaurine or iodoacetate, were left at $37^{\circ}$ for the longer periods indicated and then also cooled to $0^{\circ}$. The final pantothenate contents of the reaction mixtures suggest that the inhibition of pantothenate metabolism by pantoyltaurine occurs almost im. mediately, both in the case of streptococci and of $C$. diphtheriae. Taking the accuracy of pantothenate assay in the experiments of Table 1 as $\pm 0.1 \mathrm{~m} \mu \mathrm{mol}$., and assuming the inhibitions by 100 or 1000 equivalents of pantoyltaurine to be complete when they commence, then inhibition occurs within $2 \mathrm{~min}$. If the inhibitory action is gradual, then its onset is within an even shorter period.

The effect of 10 equivalents of pantoyltaurine, when initially present in the reaction mixture, is to inhibit pantothenate inactivation by $64 \%$ (Fig. 1B). If this degree of inhibition is assumed to take place immediately on the addition of this quantity of pantoyltaurine to suspension A (Table 1), 2.45 $\mathrm{m} \mu \mathrm{mol}$. of pantothenate would be inactivated at the end of the experimental period. This agrees well with the $2 \cdot 35 \mathrm{~m} \mu \mathrm{mol}$. observed. 
The promptness of response to removal of pantoyltaurine. This was investigated by first exposing two similar portions of a streptococcal suspension to pantothenate in reaction mixtures (Table 2) in which pantothenate inactivation could proceed

Table 2. Reversible nature of pantoyltaurine inhibition of pantothenate metabolism

Vessels contained the reaction mixture of Table 1 without casein and with pantothenate, $11.5 \mathrm{~m} \mu \mathrm{mol}$. and the group $\mathrm{G}$ streptococci (dry weight, 5.1 mg.). Vessels $(a)$ and (b) comprise the first part of the experiment, after which their contents were centrifuged, the supernatants kept for pantothenate assay, and the deposited organisms transferred to vessels $(c)$ and $(d)$.

Vessel $\begin{gathered}\text { Pantoyl- } \\ \text { taurine } \\ \text { content of } \\ \text { vessels } \\ (\mu \mathrm{mol} .)\end{gathered}$

(a) 0

(b) $\quad 0.5$

(c) 0

(d) 0
Pantothenate inactivated in equal periods of time

(approx. $60 \mathrm{~min}$.) ( $\mathrm{m} \mu \mathrm{mol}$.

8.0

$2 \cdot 1$

$6 \cdot 8$ $7 \cdot 0$

rapidly, but to one of which was added an excess of pantoyltaurine. This greatly inhibited pantothenate inactivation of the latter suspension ( $b$, Table 2). The organisms were separated from each reaction mixture by centrifuging, but were not washed. They were then transferred to vessels which contained reaction mixtures similar to the original one, but which were both without pantoyltaurine. The mixtures were placed in a thermostat at $37^{\circ}$ within $2 \frac{1}{2}$ min. after the addition of the organism, and the pantothenate inactivation in a second period was determined. All the manipulations between the two periods of pantothenate metabolism occupied 40 min., during all of which the organisms of $(b)$ were either in the presence of their original pantoyltaurine + pantothenate mixture, or in that of panto. thenate whose metabolism was determined in the second part of the experiment.

It is seen from Table $2(c, d)$ that the previously inhibited organisms fully recovered their ability to inactivate pantothenate. Assuming again in assay an accuracy of $0.1 \mathrm{~m} \mu \mathrm{mol}$. of pantothenate, recovery was within 2 min.
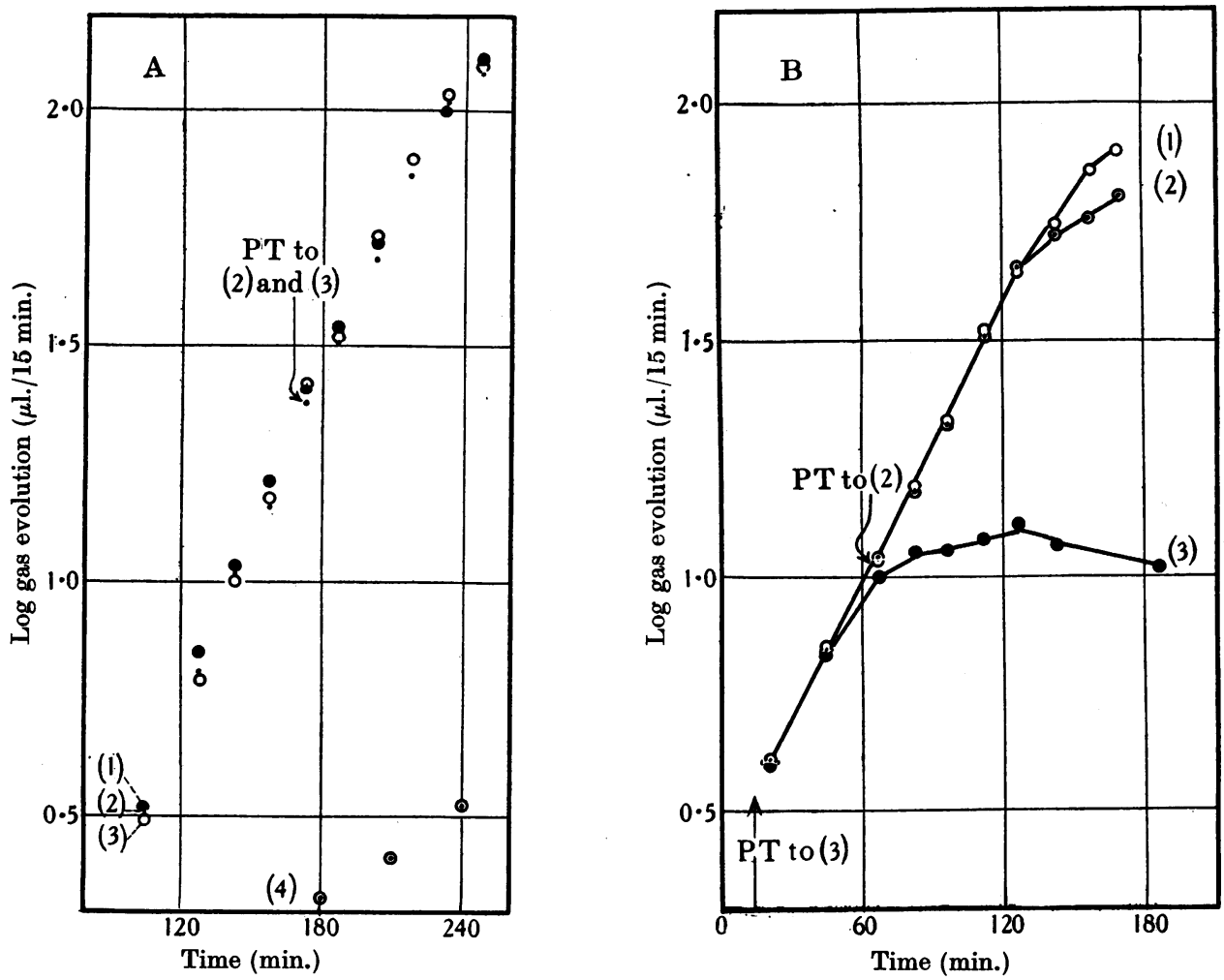

Fig. 2. Delayed action of pantoyltaurine on the course of streptococcal growth. (A) With pantothenate, $3 \cdot 3 \times 10^{-6} \mathrm{M}$; (1) without addition of inhibitor; (2) with pantoyltaurine (PT) making a final concentration of $3.3 \times 10^{-3} \mathrm{M}$ added at 170 min.; (3) with pantoyltaurine to $3.3 \times 10^{-4} \mathrm{M}$ added at $170 \mathrm{~min}$.; (4) with inoculum added to pantothenate, $3.3 \times 10^{-6} \mathrm{M}$ and pantoyltaurine, $3.3 \times 10^{-4} \mathrm{M}$. (B) A different inoculum with pantothenate, $2 \times 10^{-6} \mathrm{M}$; (1) uninhibited; (2) with pantoyltaurine to $10^{-3} \mathrm{M}$ added at $75 \mathrm{~min}$. and (3) with the same addition at $15 \mathrm{~min}$. 


\section{Promptness of action of pantoyltaurine and pantothenate on streptococcal growth}

Pantoyltaurine and normally growing cultures. The initial presence of pantoyltaurine in concentrations 20 or more times those of the pantothenate, in media whose concentration of pantothenate approximated to that of the present experiments, was found (McIlwain, 1944) to increase the length of the lag period of cultures and to decrease the velocity of their subsequent growth. A culture inhibited in this manner by a 70-fold preponderance of pantoyltaurine is shown by the points (series 4) of Fig. $2 \mathrm{~A}$ (points (series 1) show an uninhibited control). The majority of the present experiments differ from that of series (4), Fig. $2 \mathrm{~A}$, in that uninhibited growth is initiated in the presence of pantothenate, and pantoyltaurine added during growth. Such addition was found to have little if any initial effect upon the course of growth even when large excesses of pantoyltaurine were used. In the experiment of Fig. 2A, a 100- or 1000-fold preponderance of pantoyltaurine showed no effect within $1 \frac{1}{2} \mathrm{hr}$. after its addition; growth continued at the same rate as before the addition and at the same rate as in the uninhibited culture. At the end of the experiment the population was nearly the maximal size obtainable in the medium studied, and had increased 5 -fold in the presence of the inhibitor. The addition of pantoyltaurine at earlier stages in the growth of cultures is shown in Fig. 2B. A 500-fold preponderance of pantoyltaurine added $15 \mathrm{~min}$. after inoculation caused inhibition of growth within $60 \mathrm{~min}$. after the addition; when added $75 \mathrm{~min}$. after inoculation, inhibition was noticeable $75 \mathrm{~min}$. later. The uninhibited cultures of Figs. $2 \mathrm{~A}$ and $2 \mathrm{~B}$ were chosen to differ in length of lag and rate of growth, but they agree in showing considerable delay in the action of the drug. The two other cultures examined (not described in detail) showed similar delays, such that up to three generations of cells may elapse between the addition of pantoyltaurine and an inhibition of growth.

Pantoyltaurine and pantothenate added to pantoyltaurine-inhibited cultures. Reasons for carrying out these experiments are given in the discussion. In the experiment recorded in Fig. 3, pantoyltaurine in concentrations 50 times those of the coincident pantothenate led to an inhibited growth with at least two logarithmic phases. Pantoyltaurine sufficient to make a concentration 600 times that of the pantothenate was added at about the end of the first of these, and a retardation of growth was seen in the reading taken $15 \mathrm{~min}$. later but was such that the initial effect probably occurred within a shorter period approximating to $7 \mathrm{~min}$. It was not, however, an immediate and complete effect, but permitted the population of the culture to increase to a size 1.7 times that present at the time of addition of the larger quantity of pantoyltaurine. The effect thus occurred, on the average, within one generation of cells. The population of the final inhibited culture was less than one-third of the

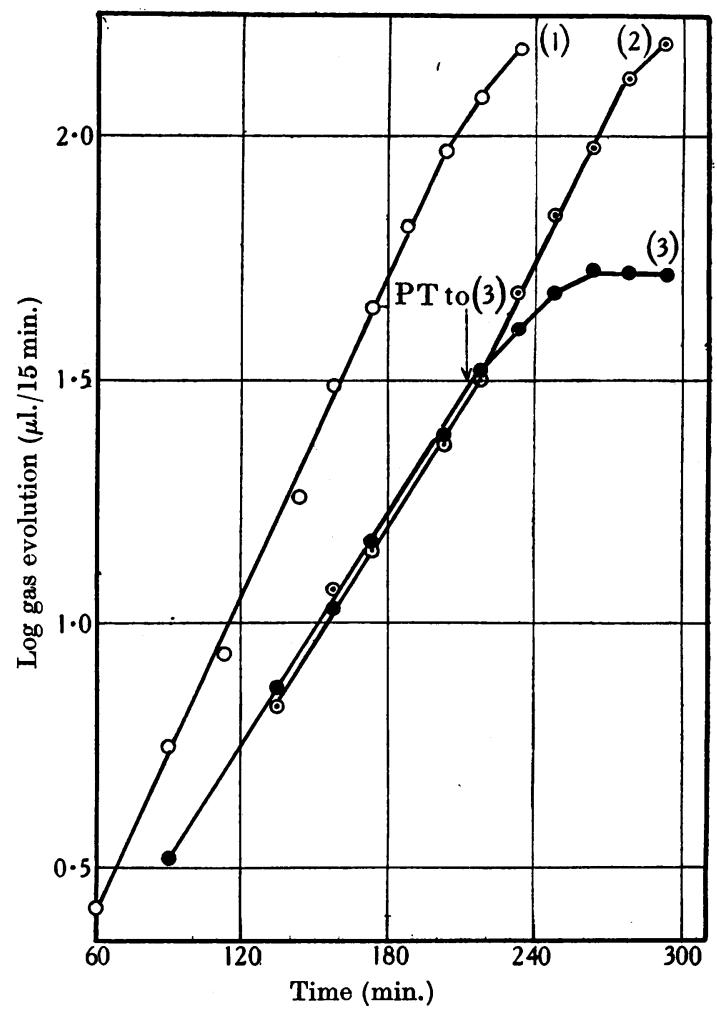

Fig. 3. A prompter action of pantoyltaurine on already inhibited streptococcal growth. (1) With pantothenate, $3.3 \times 10^{-6} \mathrm{M}$ and no inhibitor; (2) and (3) with the same quantity of pantothenate and with pantoyltaurine (PT), $1 \cdot 6 \times 10^{-4} \mathrm{M}$. Water was added to (2) and pantoyltaurine (to $3.3 \times 10^{-3} \mathrm{M}$ ) to (3) at $212 \mathrm{~min}$.

maximum size which the medium would ordinarily support. The promptness of action in this experiment is to be compared with the 4- to 8-fold increase in population which occurred before a comparable excess of pantoyltaurine showed an action on the growth of uninhibited cultures.

The cultures used to obtain the results shown in Fig. 4 all contained pantoyltaurine, and an inoculum was used whose normal growth followed a relatively long lag. Before inoculation, sufficient pantothenate was added to culture (1) to antagonize the potential inhibitory effect of the pantoyltaurine, and this quantity of pantothenate was added to cultures (2) and (3) at, respectively, 63 and 123 min. after inoculation. No addition was made to culture (4) and its growth was inhibited throughout the experi- 
ment. It can be seen that late addition of pantothenate to cultures (2) and (3) enabled them to grow but that their lag was increased from the $116 \mathrm{~min}$. of (1) to 136 and $167 \mathrm{~min}$. Thus pantothenate, also, required a measurable time to affect the growth of organisms when growth had been inhibited by pantoyltaurine. The intervals between addition of pantothenate and the beginning of the logarithmic

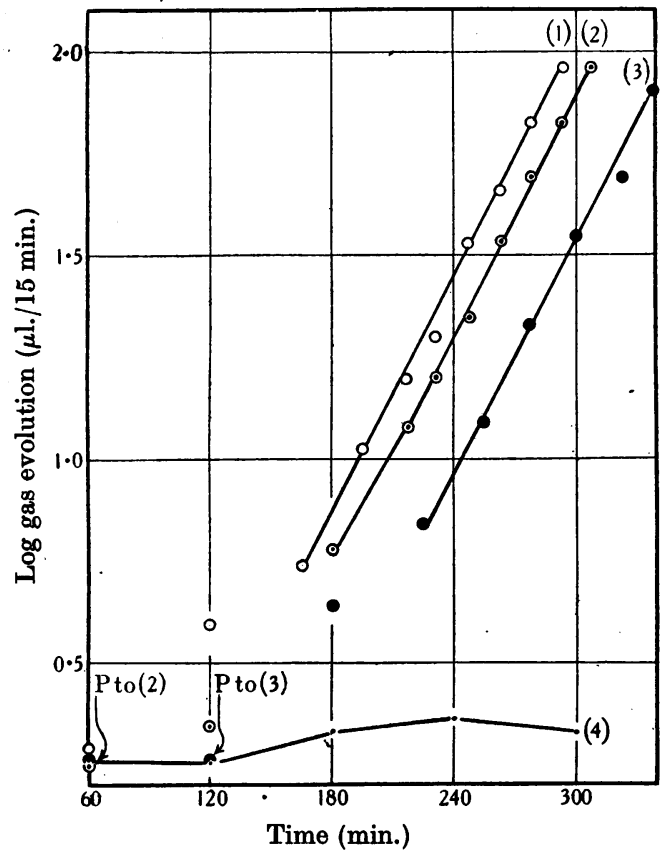

Fig. 4. Delayed effect of pantothenate in restoring pantoyltaurine-inhibited growth. (1) With, initially, pantothenate, $3.2 \times 10^{-5} \mathrm{M}$ and pantoyltaurine, $2 \times 10^{-4} \mathrm{M}$. (2), (3) and (4) with initially pantothenate, $2 \times 10^{-6} \mathrm{M}$ and pantoyltaurine, $2 \times 10^{-4} \mathrm{M}$. Pantothenate $(\mathrm{P}-0.1 \mathrm{ml}$., making a final total concentration of $3.2 \times 10^{-5} \mathrm{M}$ ) was added to (2) at $63 \mathrm{~min}$. and to (3) at $123 \mathrm{~min}$.

phase of normal growth rate in cultures (2) and (3) were 73 and $44 \mathrm{~min}$., or much less than the lag of 116 min. of culture (1); but the response to pantothenate was far from rapid even when its addition was made at a time later than that at which uninhibited growth commenced in the control, (1). It was concluded that the change from lag to logarithmic phases required certain reactions which were inhibited by excess of pantoyltaurine, and others which were independent of it.

\section{DISCUSSION}

Reasons for considering the inhibition of pantothenate inactivation by pantoyltaurine to be closely connected with the effects of the inhibitor on growth have been given previously (McIlwain \& Hughes, 1945). Nevertheless, the present study shows the two inhibitions to differ in the promptness of their onset. The following consideration of the character of the action of pantoyltaurine suggests that this finding is not inconsistent with, but can be interpreted in terms of, a causal relationship between the two inhibitions.

Previous investigations of this series have suggested that the reaction with pantothenate is associated with the production of substances necessary to bacterial growth; inhibition of the reaction thus leads to inhibition of growth. Now it has already been found (McIlwain, 1944; McIlwain \& Hughes, 1944) that the inactivation of pantothenate by streptococci is more sensitive to pantoyltaurine than is their growth. Thus a 20-fold preponderance of pantoyltaurine can decrease pantothenate inactivation to $25 \%$ of its normal rate but has only a very slight effect on growth. If its products are essential to growth, the reaction with pantothenate can therefore be carried out by the normal organisms in considerable excess of their minimal needs. The growing cultures to which pantoyltaurine was added in the experiments of p. 282 may thus be expected to have produced, before addition of the inhibitor, sufficient material for their continued growth in the presence of pantoyltaurine. The continued growth has been observed; a stable product of the expected properties has not yet been detected in solution, but may be part of the bacterial cell.

Further observations support this conception of the action of pantoyltaurine and pantothenate. (1) If the suggested reason for the delay in growth inhibition is correct, the delay should be less when less opportunity is given to the bacteria for reaction with pantothenate during their growth. This limitation should be achieved by adding an amount of pantoyltaurine having little effect on growth. As shown in Fig. 3, the slight inhibition produced by $1.6 \times 10^{-4} \mathrm{M}$-pantoyltaurine was converted relatively quickly to a complete inhibition, by the addition of further pantoyltaurine (curve 3). The second quantity of the inhibitor acted on growth within 7 min. of its addition, in comparison with the 60-75 min. elapsing before a comparable quantity affected the uninhibited growth shown in Fig. 2. (2) If products of a pantoyltaurine-sensitive reaction with pantothenate are necessary for growth, and not the mere presence of adequate pantothenate, then even though reversal of the metabolic effect of the inhibitor is prompt, time can nevertheless be expected to elapse before the effects of pantothenate, as an antagonist to the growth inhibition of pantoyltaurine, become manifest. This was concluded to be the case from examination of Fig. 4.

The present results are thus consistent with the theory that the effect of pantoyltaurine on processes 
intimately connected with pantothenate inactivation is responsible for the action of the inhibitor on growth. Evidence against the allocation of such a role to certain other metabolic effects of pantothenate has been given previously (McIlwain, 1944). The relationships between inactivation of pantothenate and its 'binding' and functioning in the cell are receiving further study.

\section{SUMMARY}

1. Low concentrations of pantoyltaurine can prevent the commencement of the growth of $\beta$-haemolytic streptococci, but when the compound is added - even in relatively high concentrations-to already growing cultures, its effect on growth is not shown until after a latent period of an hour or more. In this its action resembles that of the sulphonamides.

2. The action of pantothenate as an antagonist to growth inhibition by pantoyltaurine is also a delayed one.
3. A process of pantothenate inactivation which is connected with the action of pantoyltaurine upon growth is nevertheless inhibited promptly by pantoyltaurine both in streptococci and in Corynebacterium diphtheriae.

4. This process starts again promptly after removal of pantoyltaurine.

5. Quantitative considerations show this process to be carried out by streptococci, in their normal growth, in considerable excess of their ordinary needs. During the latent period in the action of pantoyltaurine on growth, the organisms are considered to be using materials previously made in excess.

6. When the reaction with pantothenate is limited by growth of streptococci in the presence of concentrations of pantoyltaurine which only slightly affect growth, further pantoyltaurine acts upon growth relatively promptly.

I am greatly indebted to Mr D. E. Hughes for assistance during these investigations.

\section{REFERENCES}

Henry, R. J. (1943). Bact. Rev. 7, 176.

McIlwain, H. (1944). Biochem. J. 38, 97.

McIlwain, H. \& Hawking, F. (1943). Lancet, 1, 449.
McIlwain, H. \& Hughes, D. E. (1944). Biochem. J. 38, 187.

McIlwain, H. \& Hughes, D. E. (1945). Biochem. J. 39, 133. 